\title{
Reflection of equatorial Kelvin waves at eastern ocean boundaries Part I: hypothetical boundaries
}

\author{
J. Soares ${ }^{1}$, I. Wainer ${ }^{2}$, N. C. Wells ${ }^{3}$ \\ ${ }^{1}$ Departamento de Ciências Atmosféricas, Rua do Matão 1226, Universidade de São Paulo, SP, Brazil, 05508-900 \\ 2 Departamento de Oceanografia Física, Praça do Oceanográfico 191, Universidade de São Paulo, SP, Brazil, 05508-900 \\ ${ }^{3}$ Southampton Oceanography Centre, European Way, Southampton, England, UK, SO14 3ZH
}

Received: 19 September 1997 / Revised: 26 August 1998 / Accepted: 19 October 1998

\begin{abstract}
A baroclinic shallow-water model is developed to investigate the effect of the orientation of the eastern ocean boundary on the behavior of equatorial Kelvin waves. The model is formulated in a spherical polar coordinate system and includes dissipation and nonlinear terms, effects which have not been previously included in analytical approaches to the problem. Both equatorial and middle latitude response are considered given the large latitudinal extent used in the model. Baroclinic equatorial Kelvin waves of intraseasonal, seasonal and annual periods are introduced into the domain as pulses of finite width. Their subsequent reflection, transmission and dissipation are investigated. It is found that dissipation is very important for the transmission of wave energy along the boundary and for reflections from the boundary. The dissipation was found to be dependent not only on the presence of the coastal Kelvin waves in the domain, but also on the period of these coastal waves. In particular the dissipation increases with wave period. It is also shown that the equatorial $\beta$-plane approximation can allow an anomalous generation of Rossby waves at higher latitudes. Nonlinearities generally have a small effect on the solutions, within the confines of this model.
\end{abstract}

Key words. Oceanography: general (equatorial oceanography; numerical modeling) - Oceanography: physical (eastern boundary currents).

\section{Introduction}

Observations and modeling (Enfield and Allen, 1980; Spillane et al., 1987; Paes-Sierra and O'Brien, 1989; Johnson and O'Brien, 1990) of the upper ocean suggest

Correspondence to: J. Soares that equatorial anomalies in sea level and surface temperature can propagate to middle latitudes along ocean eastern boundaries, on intra and inter-annual time scales. Of significant importance among these anomalies is the El Niño phenomenon.

Theoretical studies (Moore, 1968; Anderson and Rowlands, 1976; McCreary, 1976; Clarke, 1983, 1992; Enfield et al., 1987; Shaffer et al., 1997) have shown that the eastern boundary is an extension of the equatorial wave guide. They also point out that the reflection of low-frequency waves, incident on the boundary at the equator, should be visible at higher latitudes along the coast.

Geometrical considerations on the reflection of equatorial waves off boundaries was examined by Longuet-Higgins (1964). Low-frequency reflection from a non-meridional boundary was also studied by Schopf et al. (1981) and Grimshaw and Allen (1988) using ray theory analysis. They show the strong dependence of the coastline inclination and the frequency of the incident wave on the behavior of the wave reflection at the boundaries. These previous studies have excluded the effect of lateral viscosity and employed approximations ( $\beta$-plane, low-frequency wave, for example) to the governing equations in order to solve them analytically. Numerical solutions, however, can be obtained without these approximations. In practice, because of dissipative effects, there is a limit to the distance to which information is carried poleward by waves.

In this study we examine the importance of non linearity, viscosity and $\beta$-plane approximation when investigating the effect of the eastern boundary orientation on the reflection of baroclinic equatorial waves. Three different periods for the incoming equatorial Kelvin wave are considered: intraseasonal, seasonal and annual.

\section{Background}

The nature of the response at an eastern boundary to an incident baroclinic Kelvin wave at a single frequency $\sigma$ 
depends on the magnitude of $\sigma$ and on the latitude $(\theta)$. For the low frequencies a critical latitude, $\theta_{c}$, exists such that for $|\theta|<\theta_{c}$ the response consists of offshorepropagating Rossby waves, whereas for $|\theta|>\theta_{c}$ the waves are trapped at the coast. The magnitude of $\theta_{c}$ depends on $\sigma$ and on the angle of the coastline, $\gamma$, decreasing as $\sigma$ increases and as the orientation departs from a meridional boundary (Grimshaw and Allen, 1988). For a boundary making a constant angle $\gamma$ with due north, $\theta_{c}$ can be defined (Clarke and Shi, 1991) as:

$\left|\theta_{c}\right|=\tan ^{-1}\left[\frac{c \cos \gamma}{2 \sigma r}\right]$

where $r$ is the radius of the Earth and $c$ is the linear phase velocity.

As $\gamma$ increases, $\left|\theta_{c}\right|$ decreases and the latitude band over which Rossby waves radiates from the coast also decreases.

The strong dependence of $\left|\theta_{c}\right|$ on the coastline angle, is explained by Clarke (1992; hereafter referred as C92), in terms of potential vorticity: particles near the boundary must move parallel to it. For a given frequency and velocity amplitude, the more the boundary tilts from north-south position the less planetary vorticity change an oscillating particle will experience. When the change in planetary vorticity is sufficiently large, energy leaves the boundary as Rossby waves. Tilting the boundary favors trapped motion. In other words, inclined boundaries should be less reflective than meridional ones and the poleward coastal Kelvin wave energy flux should be increased.

\section{Model description}

A non linear, $11 / 2$ layer, reduced gravity model is used (Gill, 1982). The model equations in spherical coordinates, for the upper layer, are as follows :

$$
\begin{aligned}
& \frac{\partial u}{\partial t}+\frac{1}{r \cos \theta} \frac{\partial}{\partial \lambda}\left(\frac{u^{2}+v^{2}}{2}\right)-v(\zeta+f) \\
& \quad=-\frac{g^{\prime}}{r \cos \theta} \frac{\partial h}{\partial \lambda}+A_{H} \nabla^{2} u \\
& \frac{\partial v}{\partial t}+\frac{1}{r} \frac{\partial}{\partial \theta}\left(\frac{u^{2}+v^{2}}{2}\right)+u(\zeta+f)=-\frac{g^{\prime}}{r} \frac{\partial h}{\partial \theta}+A_{H} \nabla^{2} v \\
& \frac{\partial h}{\partial t}+\frac{1}{r \cos \theta}\left\{\frac{\partial}{\partial \lambda}(u H)+\frac{\partial}{\partial \theta}(v H \cos \theta)\right\}=0
\end{aligned}
$$

Longitude $(\lambda)$ increases eastward and latitude $(\theta)$ increases northward. As a shallow layer is being considered, $r=6.37 \times 10^{6} \mathrm{~m}$, can be taken to be a constant equal to the radius of the Earth.

Here $h(\lambda, \theta, t)$ is the interface displacement of the upper layer to the mean depth $H_{0}(\lambda, \theta) ; H(\lambda, \theta, t)$ is the total depth $\left(H=H_{0}+h\right) ; u(\lambda, \theta, t)$ and $v(\lambda, \theta, t)$ are the horizontal components of the depth averaged velocity vector $\bar{v}(\lambda, \theta, t)$ in the $\lambda$ and $\theta$ directions, respectively; $t$ is the time. Reduced gravity is $g^{\prime}=g\left(\rho_{2}-\rho_{1}\right) / \rho_{2}$ where $g$ is the acceleration of gravity, $\rho_{1}$ and $\rho_{2}$ are the density of the upper and lower layers respectively. $\zeta$ is the relative vorticity in spherical coordinates. The coriolis parameter is $f=2 \Omega \sin \theta$, with $\Omega=7.292 \times 10^{-5} \mathrm{rad} / \mathrm{s}$, being the Earth's rotation. Unless otherwise stated the upper layer initial thickness is $H_{0}=200 \mathrm{~m}$ and $g^{\prime}=0.03 \mathrm{~ms}^{-2}$. In the linearized form of the reduced gravity equations the linear phase velocity, $c=\left(g^{\prime} H_{0}\right)^{1 / 2}$ can be prescribed. Here, $c$ is taken to be $2.45 \mathrm{~ms}^{-1}$. Note that in the fully non-linear form, there is no analogous phase speed parameter. Prescribing the initial thickness, however, is analogous to prescribing an initial phase speed, which does not remain constant as the model fields evolve, since the variations in $h$ can be large compared to $H_{0}$. The dissipation coefficient $\left(A_{H}\right)$ is taken to be $10^{3}$ $\mathrm{m}^{2} \mathrm{~s}^{-1}$. These values of $c$ and $A_{H}$ have been commonly used in baroclinic shallow-water models (Davey et al., 1983).

Equations.(2)-(4) were finite-differenced using an Arakawa "C" grid (Mesinger and Arakawa, 1976). The time discretization scheme used in this work follows Castro (1985) and is described in the Appendix.

A regular $1 / 4$ degree resolution is used in both zonal and meridional directions $\left(\Delta \lambda=\Delta \theta=1 / 4^{\circ}\right)$, unless otherwise stated. For the highest latitude of the numerical domain $\left(45^{\circ}\right)$ the internal Rossby radius is $24 \mathrm{~km}$ whilst the grid spacing is $17 \mathrm{~km}$. For the linear case a time step $(\Delta t)$ of $4 \mathrm{~h}$ was utilized. For the non-linear case, the Courant-Friedrichs-Lewy (CFL) condition was used only as a guide for choosing the time interval. The $\Delta t$ actually employed in the non-linear case $(\Delta t=2 \mathrm{~h})$, was suggested by a trial-and-error process.

Two important parameters associated with $A_{H}$, characterizing the offshore and alongshore viscous effects, relative to the free Kelvin wave on a linear $f$-plane, as discussed by Davey et al. (1983), were examined.

Offshore viscous effect:

$\mathrm{E}=\frac{A_{H}}{|f| R^{3} l}$

Alongshore viscous effect:

$\alpha=\frac{A_{H} l}{|f| R}$

where $R$ is the Rossby radius $(\mathrm{c} /|f|)$ and $l$ is the alongshore wave-number.

Long waves are slowed down by offshore viscous effects (Eq. 5) and short waves by longshore viscous effects (Eq. 6). Note that the offshore viscous effects increase as wavelength increases. This seems surprising at first because one expects longer waves to be less affected by viscosity. The shorter waves are shown to decay faster. The longer waves decay more slowly and the longshore decay scale is largest for long waves.

The effects of viscosity on linear free baroclinic Kelvin waves in numerical shallow-water models were explored by Hsieh et al. (1983). A model resolution parameter was defined in their study as:

$\Delta=\frac{\Delta s}{R}$

with $\Delta s$ being the offshore grid spacing. 
They also define two distinct parameter regime. A "good-resolution" regime, $\mathbf{E} / \Delta^{2} \gg 1$ and a "poor-resolution" regime, $\mathbf{E} / \Delta^{2} \ll 1$. Transition from one regime to another regime occurs when $\mathbf{E} / \Delta^{2}=1$. They have shown that in a "good-resolution" regime, the wave behavior in a numerical model is essentially identical to that in the continuous case studied by Davey et al. (1983), i.e., finite-difference effects are negligible. Within a "poor-resolution" regime, $\Delta$ becomes dominant while E becomes unimportant.

\subsection{Boundary conditions}

A modification of Orlanski's (1976) radiative boundary condition (Miller and Thorpe, 1981) was used in all open boundaries: north, south and west, excluding corners. These open boundary conditions used in the numerical model was extensively tested (see Soares, 1994) and they allow wave transmission without anomalous reflections. They are located along lines where the velocity perpendicular to that boundary is defined. Therefore, excluding the corners, there is always one dependent variable located on each open boundary: the $\mathbf{v}$ velocity component on the northern and southern boundaries and the $\mathbf{u}$ velocity component on the western boundary. The other dependent variable (layer interface displacement) was calculated using the model equations since it is actually located inside the model domain. At the corners, where there are two dependent variables (u and v), the phase velocity was set to zero. This procedure works well in this model because the phenomena generated inside the domain are certainly weak near the model corners. The waves are mainly confined to the equator and to the coast. With the present scheme, perturbations generated inside the domain are able to cross the artificial boundaries without distortion and without affecting the interior solution.

On the solid boundaries the free-slip condition was used. It assumes that the tangential velocity outside the boundary is set equal to its adjacent counterpart inside the boundary. According to Davey et al. (1983) the wave properties, particularly the longshore wave velocity, are much less dependent on $A_{H}$ when the free-slip rather than no-slip conditions are used. Numerically, $A_{H}$ helps to smooth the very high wave number numerical noise. Physically, the coefficient helps to form a viscous boundary layer which is required to fit the observations with the theory. Davey et al. (1983), for example, suggested a value of the order of $10^{3}-10^{4} \mathrm{~m}^{2} \mathrm{~s}^{1}$. They have used the fact that the onshore velocity is nonzero when $A_{H}>0$ to estimate the amount of viscosity required in their continuous wave model to achieve a realistic onshore flow; i.e., a flow comparable to the onshore flow observed in the free baroclinic coastally trapped waves off Peru.

\section{Energy diagnostics}

To better understand the dynamics of wave reflection, one needs also to consider the wave energy flux. In the context of equatorial waves, the eastward and westward energy fluxes, are due to Kelvin and Rossby waves, respectively. The poleward energy flux along the boundary is due to coastal Kelvin waves.

According to $\mathrm{C} 92$, the nondimensional ratio $r_{N}(\gamma, \sigma)$, in percentage $(\%)$, of the northward coastal Kelvin wave energy flux to the incoming equatorial Kelvin wave energy flux for a boundary making a constant angle $\gamma$ with due north is:

$r_{N}(\gamma, \sigma)=100 \cdot \frac{e \sigma}{(\beta c)^{1 / 2} \pi^{1 / 2} \cos \gamma}(\%)$

where $e=2.71828 \ldots, \beta=2.3 \times 10^{-11} \mathrm{~m}^{-1} \mathrm{~s}^{-1} \quad$ and $\pi=3.1415 \ldots$

The ratio of the southward coastal Kelvin wave energy flux to incoming energy flux is also defined as in Eq. (8). By conservation of energy, the ratio of reflected Rossby wave energy flux to incoming equatorial Kelvin wave energy flux is defined as:

$r_{W}(\gamma, \sigma)=50-\frac{200 \cdot e \sigma}{(\beta c)^{1 / 2} \pi^{1 / 2} \cos \gamma}(\%)$

where only one hemisphere was considered.

This equation indicates that Rossby wave reflection decreases with increased slant of the boundary from meridional, since as $\gamma$ increases $\left|\theta_{c}\right|$ decreases.

The reflection and transmission of waves in the simulations are evaluated in terms of energy fluxes through the three boundaries of the domain plus the energy dissipation, as shown in Fig.1.

The equation for the non linear shallow water total energy $(K+\Pi)$ can be given by:

$\frac{\partial}{\partial t}(K+\Pi)+\nabla \cdot\left[\left(g^{\prime} h+q\right) \vec{V}\right]+D=0$

The divergent operator is $\nabla \cdot \vec{F}=\frac{1}{r \cos \theta}$ $\times\left(\frac{\partial F_{\lambda}}{\partial \lambda}+\frac{\partial}{\partial \theta}\left(F_{\theta} \cos \theta\right)\right) ;$; the dissipative term is $\mathbf{D}=\mathbf{A}_{H} \mathbf{H}$ $\left(\mathbf{u} \nabla^{2} \mathbf{u}+\mathbf{v} \nabla^{2} \mathbf{v}\right)$ and $\overrightarrow{\mathbf{V}}(\lambda, \theta, \mathbf{t})=\mathbf{u H} \overrightarrow{\mathbf{i}}+\mathbf{v H} \overrightarrow{\mathbf{j}} ; q=\left(u^{2}+v^{2}\right)$ $/ 2 ; K=q H$ (kinetic energy per unit of area) and $\Pi=g^{\prime} h^{2} / 2$ (potential energy per unit of area).

Considering the region where $\lambda=\lambda_{\text {coast }}$ and $\lambda=-\lambda_{0}$ (where $\lambda_{0}$ is the longitude away from the coast) and $\theta= \pm \theta_{0}$ (where $\theta_{0}$ is the latitude in consideration). Integrating Eq. (10) over this region and averaging in time, yields:

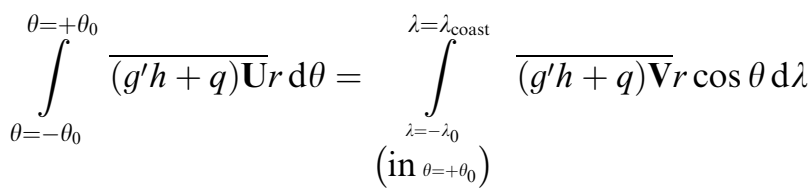

$$
\begin{aligned}
& -\int_{\substack{\lambda=-\lambda_{0} \\
\left(\operatorname{in} \theta_{\theta=+\theta_{0}}\right)}}^{\lambda=\lambda_{\text {coast }}} \overline{\left(g^{\prime} h+q\right) \mathbf{V}} r \cos \theta \mathrm{d} \lambda \\
& -\int_{\lambda=-\lambda_{0}}^{\lambda=\lambda_{\text {coast }}} \int_{\theta=-\theta}^{\theta=+\theta} \overline{\mathbf{A}_{H} \mathbf{H}\left(\mathbf{u} \nabla^{\mathbf{2}} \mathbf{u}+\mathbf{v} \nabla^{\mathbf{2}} \mathbf{v}\right)} r^{2} \cos \theta \mathrm{d} \theta \mathrm{d} \lambda
\end{aligned}
$$




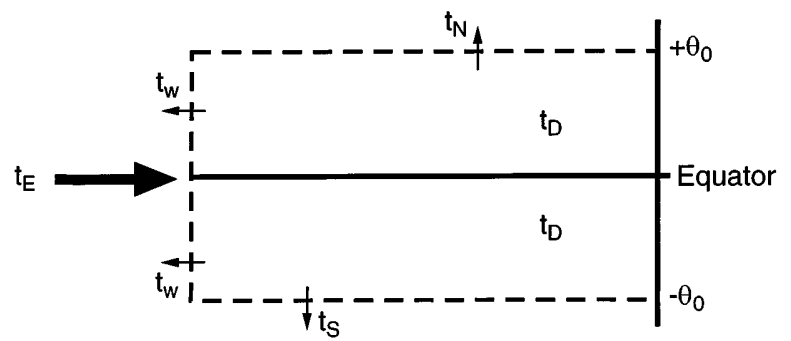

Fig. 1. Energy flux and energy dissipation generated from $\theta=\theta_{0}$ to $\theta=-\theta_{0} . t_{N}, t_{S}$ and $t_{E}$ represent, respectively, the northward, southward and the incoming energy fluxes. The energy dissipation is represented by $t_{D}$

where the overbar denotes the time average.

The equation for the linear shallow water total energy is:

$\frac{\partial}{\partial t}(K+\Pi)+\nabla \cdot\left(g^{\prime} H_{0}\right) \vec{v}+\mathbf{d}=0$

The linear dissipative term is $\mathbf{d}=\mathbf{A}_{H} \mathbf{H}_{\mathbf{0}}\left(\mathbf{u} \nabla^{2} \mathbf{u}+\mathbf{v} \nabla^{2} \mathbf{v}\right)$ and $\overrightarrow{\mathbf{v}}(\lambda, \theta, \mathbf{t})=\mathbf{u} \overrightarrow{\mathbf{i}}+\mathbf{v} \overrightarrow{\mathbf{j}} ; K=H_{0}\left(\mathbf{u}^{2}+\mathbf{v}^{2}\right) / 2$ (kinetic energy per unit of area).

Integrating Eq. (12) yields:

$$
\begin{aligned}
& \int_{\theta=-\theta_{0}}^{\theta=+\theta_{0}} \overline{\mathrm{g}^{\prime} h \mathbf{H}_{0} \mathbf{u}} \mathrm{r} \mathrm{d} \theta=\int_{\substack{\lambda=-\hat{\lambda}_{0} \\
\left(\text { in }_{\theta=+\theta_{0}}\right)}}^{\lambda=\lambda_{\text {cooast }}} \overline{\mathrm{g}^{\prime} h \mathbf{H}_{0} \mathbf{v}} \mathrm{r} \cos \theta \mathrm{d} \lambda \\
& -\int_{\lambda=-\lambda_{0}}^{\lambda=\lambda_{\text {coast }}} \overline{g^{\prime} h \mathbf{H}_{0}} \mathbf{v} \mathrm{r} \cos \theta \mathrm{d} \lambda \int_{\lambda=\lambda_{0}}^{\lambda=\lambda_{\text {coast }}} \\
& \text { (in }{ }_{\theta=+\theta_{0}} \text { ) } \\
& -\int_{\lambda=-\lambda_{0}}^{\lambda=\lambda_{\max }} \int_{\theta=-\theta_{0}}^{\theta=+\theta_{0}} \overline{\mathbf{A}_{\mathrm{H}} \mathbf{H}_{0}\left(\mathbf{u} \Delta^{2} \mathbf{u}+\mathbf{v} \Delta^{2} \mathbf{v}\right)} r^{2} \cos \theta \mathrm{d} \theta \mathrm{d} \lambda
\end{aligned}
$$

If $\lambda_{0}, \theta_{0} \rightarrow \infty$ in Eqs. (11) and (13) means that the net energy flux toward the boundary is equal to the flux outward along the boundary minus the dissipation of energy into the domain.

The energy flux outward along the boundary is due to northward $\left(t_{N}\right)$ and southward $\left(t_{S}\right)$ coastal Kelvin waves. The net energy flux $(t)$ toward the boundary is given by the difference between the energy fluxes due to the incoming equatorial Kelvin wave $\left(t_{E}\right)$ and due to the reflected Rossby waves $\left(t_{W}\right)$. Therefore: $t_{E}-t_{W}$ $=t_{N}-t_{S}-t_{D}$; where $t_{D}$ is the energy dissipation. It is assumed that the energy flux is positive to the north and to the east directions.

\subsection{Northward energy flux}

The northward energy flux, across a given latitude $+\theta_{0}$ can be obtained by:

$t_{N(\text { linear })}=\sum_{\lambda=-\lambda_{0}}^{\lambda=\lambda_{\text {coast }}} \overline{g^{\prime} h \mathbf{H}_{0} \mathbf{v} r} \cos \theta_{0} \Delta \lambda$

$$
t_{N(\text { non-linear })}=\sum_{\lambda=-\lambda_{0}}^{\lambda=\lambda_{\text {coast }}} \overline{\left(g^{\prime} h+q\right) \mathbf{V}} r \cos \theta_{0} \Delta \lambda
$$

\subsection{Net energy flux in the east-west direction}

The net energy flux toward the boundary, across a given longitude $-\lambda_{0}$, is:

$$
\begin{gathered}
t_{\text {(linear) }}=t_{E(\text { linear })}-t_{W(\text { linear })}=\sum_{\theta=-\theta_{0}}^{\theta=+\theta_{0}} \overline{g^{\prime} h \mathbf{H}_{0} \mathbf{u}} r \Delta \theta \\
t_{(\text {non-linear })}=t_{E(\text { non-linear })}-t_{W(\text { non-linear })} \\
=\sum_{\theta=-\theta_{0}}^{\theta==+\theta_{0}} \overline{\left(g^{\prime} h+q\right) \mathbf{U} r \Delta \theta}
\end{gathered}
$$

The direct determination of $t_{E}-t_{W}$, however, is computationally expensive because the propagation speed of the westward Rossby waves is a strong function of the latitude, varying from $\sim 0.8 \mathrm{~m} / \mathrm{s}$ at the equator to zero at the poles. It could take years of numerical integration for all the Rossby waves generated at the eastern boundary, to reach the western side of the domain. To save computer time, first $t_{E}$ is calculated and then $t_{W}$ is inferred by energy conservation considerations. The energy input in the experiment is only due to the baroclinic equatorial Kelvin wave. Consequently before the reflection of this wave at the eastern coast, the eastward energy flux through a given longitude of the numerical domain corresponds to the value of $t_{E}$. It can be assumed that the reflected Rossby wave energy $\left(t_{W}\right)$, for the Northern Hemisphere, is given by $t_{W} / 2=t_{E} / 2-t_{N}-t_{D} / 2$.

\subsection{Energy dissipation}

The dissipation of energy, up to a given latitude $+\theta_{0}$, can be calculated as:

$$
\begin{aligned}
t_{D(\text { linear })}= & -\sum_{\lambda=-\lambda_{0}}^{\lambda=\lambda_{\text {coast }}} \sum_{\theta=0}^{\theta=+\theta_{0}} \\
& \times \overline{\mathbf{A}_{H} \mathbf{H}_{0}\left(\mathbf{u} \nabla^{2} \mathbf{u}+\mathbf{v} \nabla^{2} \mathbf{v}\right)} r^{2} \cos \theta \Delta \theta \Delta \lambda
\end{aligned}
$$

$$
\begin{aligned}
& t_{D(\text { non-linear })} \\
& =-\sum_{\lambda=-\lambda_{0}}^{\lambda=\lambda_{\text {coast }}} \sum_{\theta=0}^{\theta=+\theta} \overline{\mathbf{A}_{H} \mathbf{H}\left(\mathbf{u} \nabla^{\mathbf{2}} \mathbf{u}+\mathbf{v} \nabla^{\mathbf{2}} \mathbf{v}\right)} r^{2} \cos \theta \Delta \theta \Delta \lambda
\end{aligned}
$$

Expressions (14), (15), (18) and (19) consider only the Northern Hemisphere. Changing $\theta=+\theta_{0}$ for $\theta=-\theta_{0}$ in those expressions gives the Southern Hemisphere fluxes. In all the numerical experiments performed in this work, the energy input is always considered for the both hemispheres and the poleward, westward and energy dissipation are always calculated for the Hemisphere in consideration. 


\section{Experiments}

A series of experiments were designed to study the free wave solutions generated by low frequency wave reflection at meridional and inclined eastern boundaries. In past works, the numerical investigation of this problem has mainly involved the forced solutions of the equations. In this study we focus on the reflection at an eastern boundary of a pulse of finite width (Kelvin wave) propagating eastward along the equator.

The mechanism for the generation of the incoming equatorial Kelvin wave is not included in the study but the Kelvin wave could, for example, be set up at the western boundary by planetary waves.

Three different wave periods $(\mathrm{T}=60, \mathrm{~T}=180$ and $\mathrm{T}=360$ days) are considered. Results for $\mathrm{T}=60$ days are presented for the linear and non-linear scenario at different boundary inclinations. The linear experiment uses $\gamma=0^{\circ}(\mathrm{C} 0), \gamma=40^{\circ} \quad(\mathrm{C} 40), \gamma=50^{\circ}$ (C50) and $\gamma=60^{\circ}(\mathrm{C} 60)$. For $\mathrm{T}=180$ days, $\mathrm{T}=360$ days and for the non-linear case of $\mathrm{T}=60$ days only the results for $\mathrm{C} 0$ and $\mathrm{C} 60$ are discussed. The results for the other cases (C40 and C50) lie between the results of $\mathrm{C} 0$ and C60. The experiments performed are summarized in Table 1 .

In each case it is just the eastern boundary of the Northern Hemisphere which is inclined. Therefore, only the Northern Hemisphere is considered for the calculation of the energy fluxes. The southward energy fluxes and the northward energy flux generated at $\gamma=0^{\circ}$ are symmetric in relation to the equator; i.e., $\left|t_{S}\left(0^{\circ}, \mathrm{T}\right)\right|$ $=\left|t_{S}\left(40^{\circ}, \mathrm{T}\right)\right|=\left|t_{S}\left(50^{\circ}, \mathrm{T}\right)\right|=\left|t_{S}\left(60^{\circ}, \mathrm{T}\right)\right|=\left|t_{N}\left(0^{\circ}, \mathrm{T}\right)\right|$.

The numerical model is integrated for a time long enough to allow for the coastal Kelvin wave, generated by the equatorial wave to leave the domain.

To investigate the wave behavior in the present numerical model, a table based on Hsieh et al. (1983) was built. Table 2 shows the parameters regime values applied for the highest latitude of the numerical domain used in the experiments. For the intraseasonal $(T=60$ days) experiment $\theta_{\max }=20^{\circ}$ and for the seasonal $(\mathrm{T}=180$ days $)$ and annual $(\mathrm{T}=360$ days $)$ experiments $\theta_{\max }=45^{\circ}$. The Rossby radius is reasonably well resolved by the model grid, $\Delta<1$, and the numerical model is in "good resolution" regime, $\mathbf{E} / \Delta^{2} \gg 1$. Therefore, the wave behavior is expected to be similar to that in the continuous case.

The offshore viscous effect (E) increases considerably as both the wavelength and the latitude increase. The increase in $\mathbf{E}$ causes a large increase in decay rate and a decrease in velocity as a wave travels poleward. The use of free-slip boundary, however, prevents the drop in speed being very severe. The alongshore coefficient $(\alpha)$ is very small in all the experiments.

\subsection{Initial condition}

A pulse of finite width, used as surrogate to a baroclinic equatorial Kelvin wave (Fig. 2), is utilized as an initial condition of the western, southern and northern unbounded ocean. The maximum amplitude of the initial disturbance was taken as $30 \mathrm{~m}$. From the hydrostatic balance this depression of the interface, implies a $10 \mathrm{~cm}$ surface elevation (considering $H_{0}=200 \mathrm{~m}, \rho_{2}=1060$ and $\rho_{1}=1025 \mathrm{Kg} \mathrm{m}^{-3}$ ). The Kelvin wave energy is equipartitioned between potential and kinetic energy thus both height and velocity information are prescribed simultaneously from initial conditions (see Anderson and Moore, 1985). The wave meridional structure is assumed to be a Gaussian function of latitude centered on the equator. Only half a wavelength of the equatorial Kelvin wave is used in the experiments. The western side of the basin is considered an open boundary in order to avoid reflection of the westward long Rossby waves. Therefore, the whole solution of the experiments can be regarded as the result of the incident Kelvin wave reaching the eastern boundary.

To avoid the equatorial Kelvin wave reaching the eastern coast before the calculation of $t_{E}$, The longitudinal extent of the basin is doubled, with the initial field placed in the left hand side of the domain. It assures that only the eastward energy flux is determined and not a combination of eastward and westward energy fluxes.

\subsection{Intraseasonal variability}

An equatorial Kelvin wave of intraseasonal period is introduced into the numerical model and the reflective properties of the eastern boundary as a function of inclination angle are investigated. A 60 day wave period was chosen because fluctuations of 40-60 day periods have been found in sea level records of the Pacific Ocean (Luther, 1980) and could be related to ENSO (Madden and Julian, 1971, 1972; Enfield, 1987; McPhaden and Taft, 1988).
Table 1. Linear and non linear experiments performed for different values of coast inclination $(\gamma)$, wave period (T60, T180 and T360 days), dissipation coefficient $\left(A_{H}\right)$ and grid spacing $(\Delta \mathrm{s})$ are marked

\begin{tabular}{|c|c|c|c|c|c|c|}
\hline & \multicolumn{5}{|c|}{ Linear } & \multirow{3}{*}{$\begin{array}{l}\text { Non linear } \\
A_{H}=10^{3} \mathrm{~m}^{2} \mathrm{~s}^{-1} \\
\Delta s=1 / 4^{\circ} \\
\mathrm{T} 60\end{array}$} \\
\hline & \multicolumn{3}{|c|}{$\begin{array}{l}A_{H}=10^{3} \mathrm{~m}^{2} \mathrm{~s}^{-1} \\
\Delta s=1 / 4^{\circ}\end{array}$} & \multirow{2}{*}{$\begin{array}{l}A_{H}=500 \mathrm{~m}^{2} \mathrm{~s}^{-1} \\
\Delta s=1 / 8^{\circ} \\
\mathrm{T} 60\end{array}$} & \multirow{2}{*}{$\begin{array}{l}A_{H}=0 \mathrm{~m}^{2} \mathrm{~s}^{-1} \\
\Delta s=1 / 4^{\circ} \\
\mathrm{T} 60\end{array}$} & \\
\hline & T60 & $\mathrm{T} 180$ & T360 & & & \\
\hline $\begin{array}{l}\gamma=0^{\circ} \\
\gamma=40^{\circ} \\
\gamma=50^{\circ} \\
\gamma=60^{\circ}\end{array}$ & $\begin{array}{l}X \\
X \\
X \\
X\end{array}$ & X & $X$ & $X$ & $X$ & $X$ \\
\hline
\end{tabular}


Table 2. Non dimensional parameters associated with the viscosity and to finite-differencing

\begin{tabular}{lllll}
\hline & $\begin{array}{l}\alpha, \text { alongshore } \\
\text { viscous effect } \\
\text { (given by Eq. 6) }\end{array}$ & $\begin{array}{l}\text { E, offshore } \\
\text { viscous effect } \\
\text { (given by Eq. 5) }\end{array}$ & $\begin{array}{l}\Delta, \text { model } \\
\text { resolution } \\
\text { (given by Eq. 7) }\end{array}$ & $\begin{array}{l}\mathbf{E} / \Delta^{2}: \\
\text { regime } \\
\text { resolution }\end{array}$ \\
\hline $\begin{array}{l}\mathrm{T} 60 ; \Delta S=1 / 8^{\circ} ; \\
A_{H}=500 \mathrm{~m}^{2} \mathrm{~s}^{-1}\end{array}$ & $1.010^{-4}$ & 0.17 & 0.27 & 2.33 \\
$\begin{array}{l}\mathrm{T} 60 ; \Delta S=1 / 4^{\circ} ; \\
A_{H}=10^{3} \mathrm{~m}^{2} \mathrm{~s}^{-1}\end{array}$ & $2.010^{-4}$ & 0.34 & 0.53 & 1.21 \\
$\begin{array}{l}\mathrm{T} 180 ; \Delta S=1 / 4^{\circ} ; \\
A_{H}=10^{3} \mathrm{~m}^{2} \mathrm{~s}^{-1}\end{array}$ & $7.010^{-5}$ & 4.5 & 0.8 & 6.6 \\
$\begin{array}{l}\mathrm{T} 360 ; \Delta S=1 / 4^{\circ} ; \\
A_{H}=10^{3} \mathrm{~m}^{2} \mathrm{~s}^{-1}\end{array}$ & $3.010^{-5}$ & 9.0 & 0.8 & 13.2 \\
\hline
\end{tabular}

The model is run in this case from $20^{\circ} \mathrm{N}$ to $20^{\circ} \mathrm{S}$ and extends $70^{\circ}$ in longitude. It is integrated for 90 days, sufficient time to allow the coastal wave generated by the initial pulse (Kelvin wave) to leave the domain.

5.2.1 Linear response. The Kelvin response for $\mathrm{C} 0$ is shown in Fig. 3 while Fig. 4 shows the response for C60. Latitude versus longitude plots of the linear upper layer anomaly are displayed at six successive intervals of 15 days. An upward movement of the interface produces a negative height anomaly, indicating upwelling.

When the baroclinic equatorial Kelvin wave reaches the coast, part of the incident energy continues poleward into either hemisphere as coastally trapped Kelvin waves (deepened thermocline along the coastline in Figs. 3 and 4). The remainder of the energy is reflected in the form of westward Rossby waves. The Rossby waves are evident in the figures as a closed contour, moving to the west from the coast, with a deeper thermocline at their center.

Figure 5 shows the nondimensional linear energy flux coefficients (in \% ) generated at $\mathrm{C} 0, \mathrm{C} 40, \mathrm{C} 50$ and $\mathrm{C} 60$, respectively. The coefficients, $r_{N}(\gamma, \mathrm{T}), r_{W}(\gamma, \mathrm{T})$ and $r_{D}(\gamma, \mathrm{T})$ are, respectively, the ratios of northward coastal

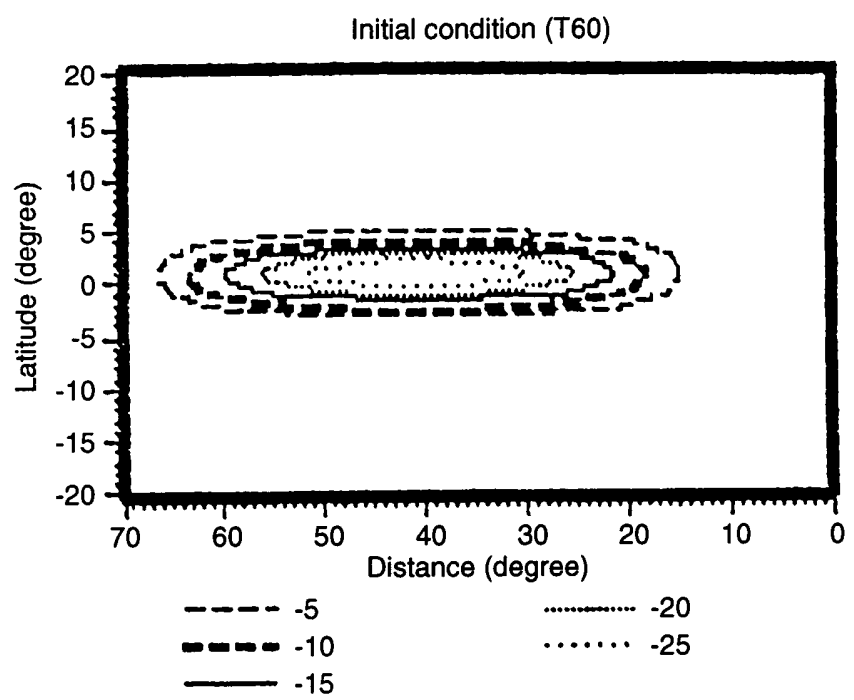

Fig. 2. Initial interface anomaly (in $\mathrm{m}$ ) used as initial condition (baroclinic equatorial Kelvin wave). $\mathrm{T}=60$ days. The zonal current is proportional to the meridional gradient of sea-level interface
Kelvin wave, of reflected Rossby wave and of the dissipation of energy fluxes to the total incoming equatorial Kelvin wave energy flux $\left(r_{N}=t_{N} / t_{E}, r_{W}=t_{W} / t_{E}\right.$ and $\left.r_{D}=t_{D} / t_{E}\right)$. For each hemisphere $\left|r_{N}\right|$ (or $\left.\left|r_{S}\right|\right)+\left|r_{W}\right|+\left|r_{D}\right|=50 \%$. The coefficients are calculated at every $2^{\circ}$ of latitude and integrated over 90 days. $r_{N}$ represents the northward energy flux crossing a given latitude and not the northward energy flux generated at that particular latitude. Similarly, $r_{W}$ and $r_{D}$ represent, respectively, the reflected and the dissipated energy fluxes up to a given latitude.

Table 3 shows $\theta_{c}$ for each $\gamma$ considered. It can be noted that $\theta_{c}$ decreases as $\gamma$ increases. According to Fig. 5 above $\theta_{c}$ (indicated in Fig. 5 by an arrow) the westward energy fluxes do not change significantly with latitude, but the poleward fluxes show an important latitudinal decrease. Theoretically above $\theta_{c}$ both energy fluxes, $r_{W}$ and $r_{N}$, should be constant with latitude.

The poleward energy flux decline, observed in Fig. 5, can be explained in terms of dissipative processes. The dissipation of energy increases with both the increase of latitude and with the proximity of the coast (lateral friction). In these experiments the $\theta_{c}$ values are relatively near the equator and therefore the coastal Kelvin waves are important in a large area of the domain. Hence most of the energy lost by dissipation in the domain is taken from the coastal Kelvin waves which results in a reduction of the poleward energy flux. The westward Rossby waves are less affected by dissipation.

To clarify the importance of the dissipation of energy in the domain, the same experiments are undertaken for the inviscid case $\left(A_{H}=0\right.$, in the numerical model). Figure 6 shows the numerical energy flux coefficients for this case. The energy flux coefficients for $A_{H} \neq 0$ are also plotted for comparison. In the inviscid case, above $\theta_{c}$ the energy fluxes are latitudinally constant, as expected. The numerical values of $r_{N}$ (solid line) and $r_{W}$ (dashed line) are $\sim 24 \%$ and $\sim 26 \%$, respectively.

The analytical values of $r_{N}$ and $r_{W}$, in the Northern Hemisphere, can be obtained using Eqs. (8) and (9). For an equatorial Kelvin wave of 60 day period incident at a meridional boundary, the analytical values $r_{N}$ and $r_{W}$ are, respectively, $\sim 24 \%$ and $\sim 26 \%$. It shows that the coefficients generated by the inviscid numerical model are in excellent agreement with the analytical coefficient values. The analytical and numerical values of $r_{W}$ as a 

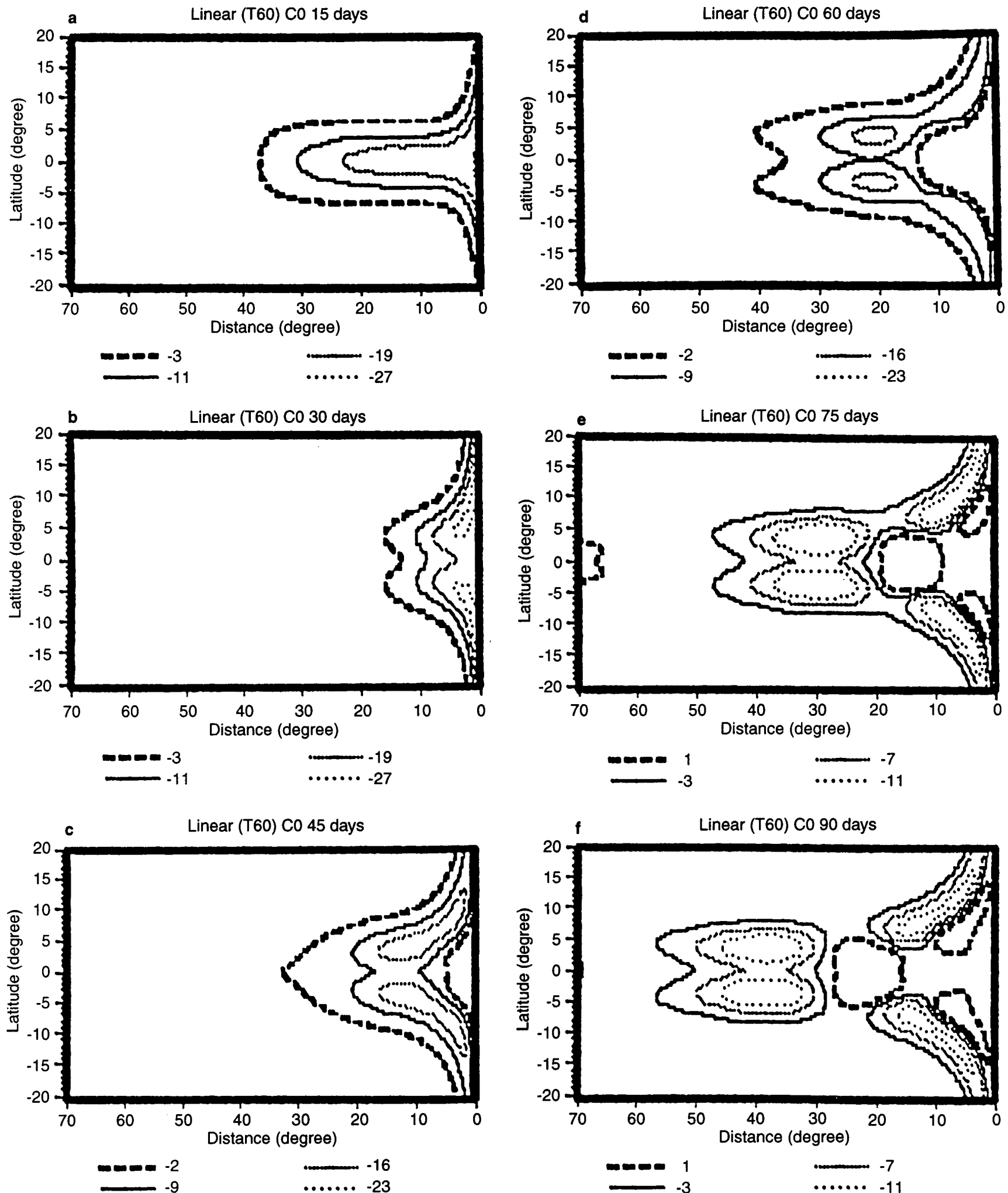

Fig. 3a-f. Sequential patterns of the linear interface anomaly (in m), at (a) 15, (b) 30, (c) 45, (d) 60, (e) 75 and (f) 90 days. C0. T=60 days

function of the different eastern boundary slopes are shown in Table 4, when $A_{H}=0$ analytical and numerical values of $r_{W}$ are the same. For all the slanted boundaries considered, the numerical values of $r_{W}$ are larger than the analytical ones. This deviation between the numer- ical and theoretical $r_{W}$ values increases as the inclination of the coast increases.

According to Hsieh et al. (1983), the wave behavior in numerical models can be very different from that in the real ocean for the following reasons: (1) the use of 

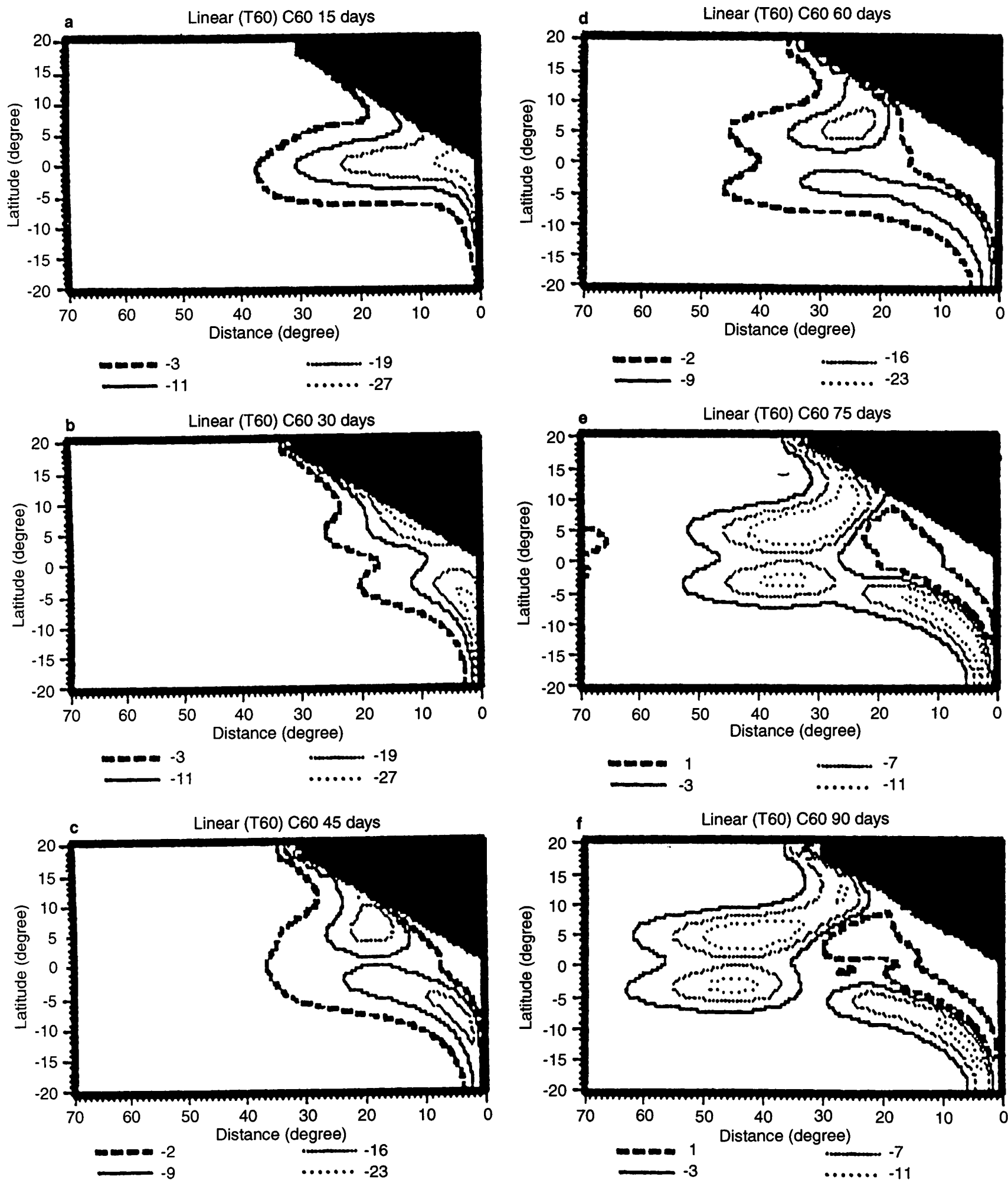

Fig. 4a-f. Sequential patterns of the linear interface anomaly (in m), at (a) 15, (b) 30, (c) 45, (d) 60, (e) 75 and (f) 90 days. C60. T =60 days

unrealistic large viscosity coefficients; (2) poor spatial resolution; and (3) boundary conditions.

In this study, boundary conditions are not a problem since they have been shown to transmit waves without any anomalous reflection (Soares, 1994). To understand the effect of (1) and (2), on the discrepancy between analytical and theoretical $r_{W}$ values, another experiment, similar to $\mathrm{C} 60$ but with a higher spatial resolution $\left(\Delta S=1 / 8^{\circ}\right.$ in both directions) and a smaller dissipation coefficient $\left(A_{H}=500 \mathrm{~m}^{2} \mathrm{~s}^{-1}\right)$ was made. This experiment is called $\mathrm{C} 60-\mathrm{H}$. The model resolution and the dissipation coefficients were changed in the same run to 

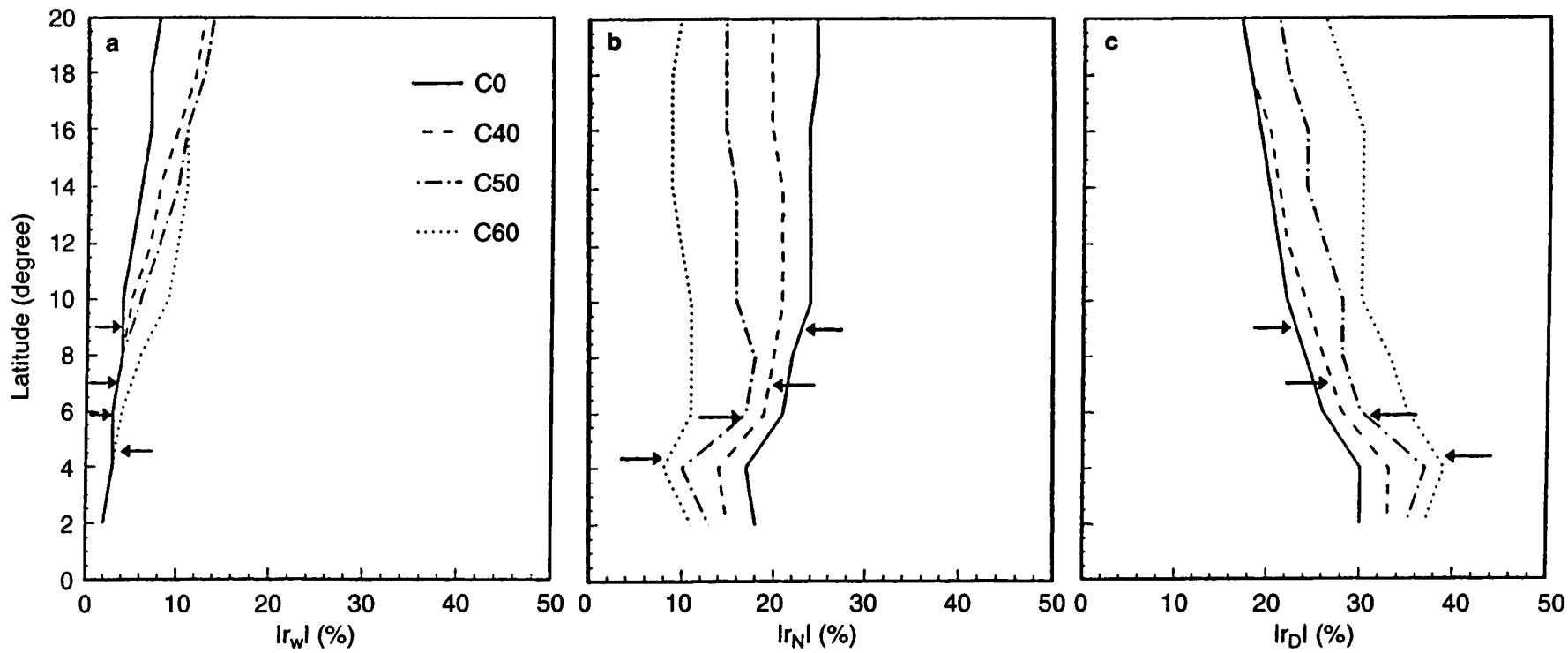

Fig. 5a-c. Linear energy flux coefficient (\%) at different latitudes $\left({ }^{\circ}\right)$. (a) $r_{W}$, (b) $r_{N}$ and (c) $r_{D}$. Solid line for C0. Dashed line for C40. Center-dashed line for C50. Dotted line for C60. The arrows indicate the critical latitude values for the investigated boundaries. $\mathrm{T}=60 \mathrm{days}$

improve the model regime. Using $A_{H}=500 \mathrm{~m}^{2} \mathrm{~s}^{-1}$ and $\Delta S=1 / 4^{\circ}$ would lead to a "poor resolution" regime $\left(\mathbf{E} / \Delta^{2}=0.6\right)$.

Figure 7 shows the westward energy flux produced by the $\mathrm{C} 60-\mathrm{H}$ experiment. The $r_{W}$ coefficients from $\mathrm{C} 60$ are also plotted, for comparison. The $\mathrm{C} 60-\mathrm{H}$ experiment produces $r_{W}$ values even larger than those generated by C60 experiment. This indicates that the large difference between the numerical and analytical westward energy fluxes with a sloping boundary is neither due to the use of unrealistic large viscosity coefficient nor to the poor spatial resolution of the numerical model. Note that the C60-H experiment is in a better "resolution regime" than the C60 case (see Table 2).

C92 uses Eq. (9) to calculate $r_{W}$ assuming that $\omega|\tan \gamma / f| \ll 1$. Table 5 displays these values for the frequency and the inclination of the coastlines used. The Coriolis parameter $(f)$ was calculated at $\theta_{c}$. According to Table 5, as $\gamma$ increases the value of $\omega|\tan \gamma / f|$ also increases and the validity of Eq. 9 becomes dubious. The comparatively larger values of $\omega|\tan \gamma / f|$ obtained for the C50 and C60 experiments could invalidate the use of Eq. (9) and be partially responsible for the large discrepancies observed between analytical and the theoretical values of the reflected energy fluxes.

From Fig. 5a, for latitudes higher than $\theta_{c}$, the values of $r_{W}$ are approximately constant with latitude in all

Table 3. Critical latitude values $\left(\theta_{c}\right)$ for $\gamma=0^{\circ}, \gamma=40^{\circ}, \gamma=50^{\circ}$ and $\gamma=60^{\circ} . \mathrm{T}=60$ days.

\begin{tabular}{ll}
\hline & $\theta_{c}\left({ }^{\circ}\right)$ \\
\hline$\gamma=0^{\circ}$ & 9.0 \\
$\gamma=40^{\circ}$ & 7.0 \\
$\gamma=50^{\circ}$ & 5.8 \\
$\gamma=60^{\circ}$ & 4.5 \\
\hline
\end{tabular}

experiments. The westward energy flux is smaller as $\gamma$ increases, in qualitative agreement with theory. This is consistent with the values of $\theta_{c}$ given in Table 3 , with westward motion favored for the more poleward values of $\theta_{c}$.

The poleward energy flux generated at $\mathrm{C} 0$, shown in Fig. 5b, is slightly smaller than that of C40 and significantly smaller than the poleward energy flux generated at both $\mathrm{C} 50$ and $\mathrm{C} 60$. The resemblance between the poleward energy fluxes for $\mathrm{C} 0$ and $\mathrm{C} 40$ can be understood by looking at Fig. 5c. The dissipation of energy increases when the coastline departs from the meridional direction. The increase in dissipa-

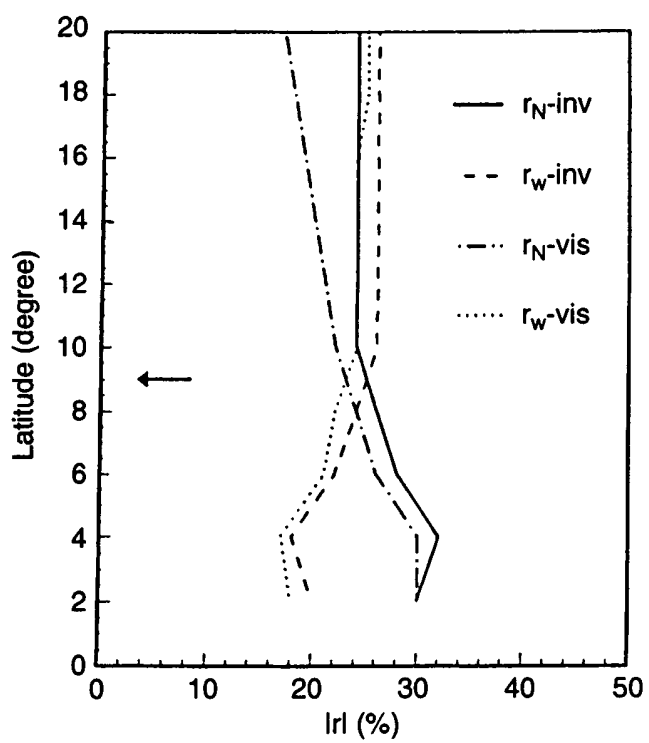

Fig. 6. Linear energy flux coefficient (in \%) at different latitudes $\left(^{\circ}\right)$. Solid and dashed lines: $r_{N}$ and $r_{W}$, respectively, for the inviscid case. Center-dashed and dotted lines: $r_{N}$ and $r_{W}$, respectively, for the dissipative experiment. $\mathrm{C} 0 . \mathrm{T}=60$ days 
Table 4. Linear westward energy flux coefficients (in $\%$ of the incident energy flux). The value in brackets is the coefficient obtained by the inviscid model. $\mathrm{T}=60$ days.

\begin{tabular}{lll}
\hline & $r_{W}$ (model) & $r_{W}$ (Eq. 9) \\
\hline$\gamma=0^{\circ}$ & $24(26)$ & 26 \\
$\gamma=40^{\circ}$ & 20 & 18 \\
$\gamma=50^{\circ}$ & 15 & 11 \\
$\gamma=60^{\circ}$ & 10 & 0.5 \\
\hline
\end{tabular}

tion, however, is not a linear function of the coastline inclination. That is, the values of $r_{D}$ at C40, C50 and C60 are comparable at higher latitudes of the domain.

In the $\mathrm{C} 40$ experiment, the increase in $r_{N}$ induced by the coastline inclination seems to be counterbalanced by the increase of energy dissipation, resulting in $r_{N}$ values similar for $\mathrm{C} 0$ and $\mathrm{C} 40$.

The dissipation of energy in the non meridional experiments (C50 and $\mathrm{C} 60)$ is not large enough to compensate for the increase of energy induced by the coastline geometry (Fig. 5), despite the fact that $r_{D}$ is larger. Therefore, the poleward energy fluxes generated at C50 and C60 are significantly larger than $r_{N}$ created at $\mathrm{C} 0$. There are two main reasons that account for the larger values of $r_{D}$. First, $\theta_{c}$ for the slanted boundary is lower than that for the meridional one. As a result, when the boundary is tilted from the north-south direction comparatively fewer Rossby waves and comparatively more coastal Kelvin waves are present in the domain. Given that the dissipative effects are larger near the coast than in the interior domain, these Kelvin waves, trapped at the coast, are more affected by the dissipation than the Rossby waves. Second, the step-like form used for approximating the tilted eastern boundary generates some numerical noise.

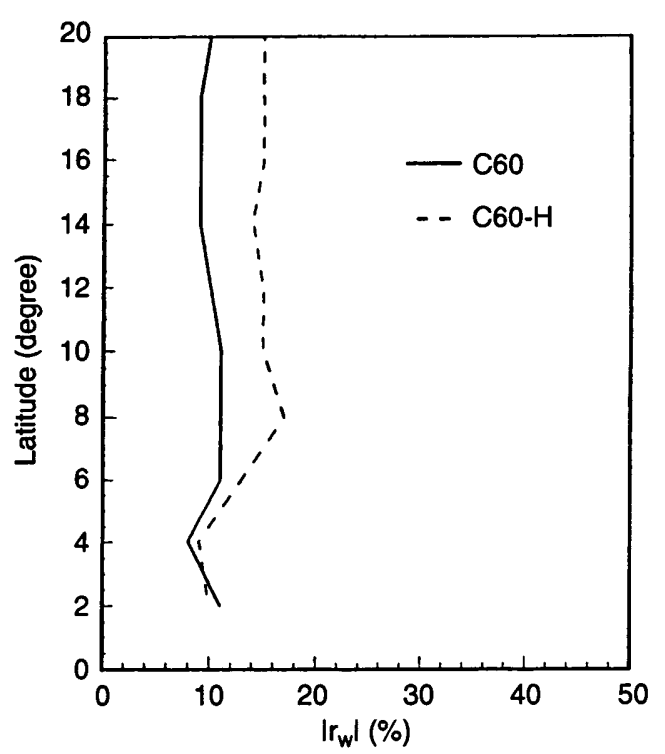

Fig. 7. Linear westward energy flux coefficient $(\%)$ at different latitudes $\left({ }^{\circ}\right)$. Solid line for $\mathrm{C} 60$ and dashed line for $\mathrm{C} 60-\mathrm{H} . \mathrm{T}=60$ days
Table 5. Values of $\omega|\tan \gamma / f|$ for different values of $\gamma$ considered in the numerical experiments. The Coriolis parameter, $f$, was calculated at the respective $\theta_{c}$ (given in Table 3). The intraseasonal frequency is $\omega=1.2 \times 10^{-6} \mathrm{~s}^{-1}$

\begin{tabular}{ll}
\hline & $\omega|\tan \gamma / f|$ \\
\hline$\gamma=40^{\circ}$ & 0.06 \\
$\gamma=50^{\circ}$ & 0.10 \\
$\gamma=60^{\circ}$ & 0.18 \\
\hline
\end{tabular}

In summary, when an equatorial Kelvin wave of intraseasonal period reaches any eastern oceanic boundary, the amount of energy transferred to poleward coastal Kelvin waves will be strongly dependent on the amount of dissipation considered in the problem. The generation of reflected Rossby waves is significantly less dependent on the dissipation utilized.

5.2.2 Non-linear response. The experiments ( $\mathrm{C} 0$ and $\mathrm{C} 60)$ are repeated using the non linear equations in order to understand the possible effects of the non linearity in low frequency wave reflection at eastern boundaries. The same pulse of finite width used as initial condition of the linear case is used for the non linear experiment.

Figure 8 shows both coefficients, linear and non linear, plotted together at different latitudes. Like the linear experiment, the non linear northward and westward energy fluxes generated at C60 are, respectively, larger and smaller than those generated at $\mathrm{C} 0$. Also the energy dissipated in $\mathrm{C} 60$ is larger than in $\mathrm{C} 0$. For the meridional boundary experiment the non linear and the linear energy flux coefficients can be roughly considered similar. For C60, the non linear westward energy flux presents a larger latitudinal variation when compared with the linear case (see Fig. 8a).

The major difference between the linear and non linear experiments for $\mathrm{C} 60$ occurs in the $r_{D}$ coefficient (Fig. 8c). Above latitude $10^{\circ}$, the dissipation of energy is considerably larger in the non linear case. Consequently, the non linear poleward energy flux is smaller when compared to the linear one (see Fig. 8b). Unlike the linear case, the non linear dissipation of energy affects not only the coastal waves but also the reflected westward Rossby waves (e.g., Fig. 8a).

\subsection{Seasonal variability}

An equatorial Kelvin wave of seasonal period $(T=180$ days) is used as the initial condition of the numerical model. Because of the increase in $\theta_{c}$ with wave period, the latitudinal extent of the model is increased to $45^{\circ} \mathrm{S}$ to $45^{\circ} \mathrm{N}$. Since the difference between linear and non linear response is small only the linear case will be discussed.

To calculate the energy fluxes, the numerical model is integrated for six months, the necessary time for the coastal Kelvin waves to leave the domain. The $\theta_{c}$ values for $\mathrm{C} 0$ and $\mathrm{C} 60$, are shown in Table 6. 

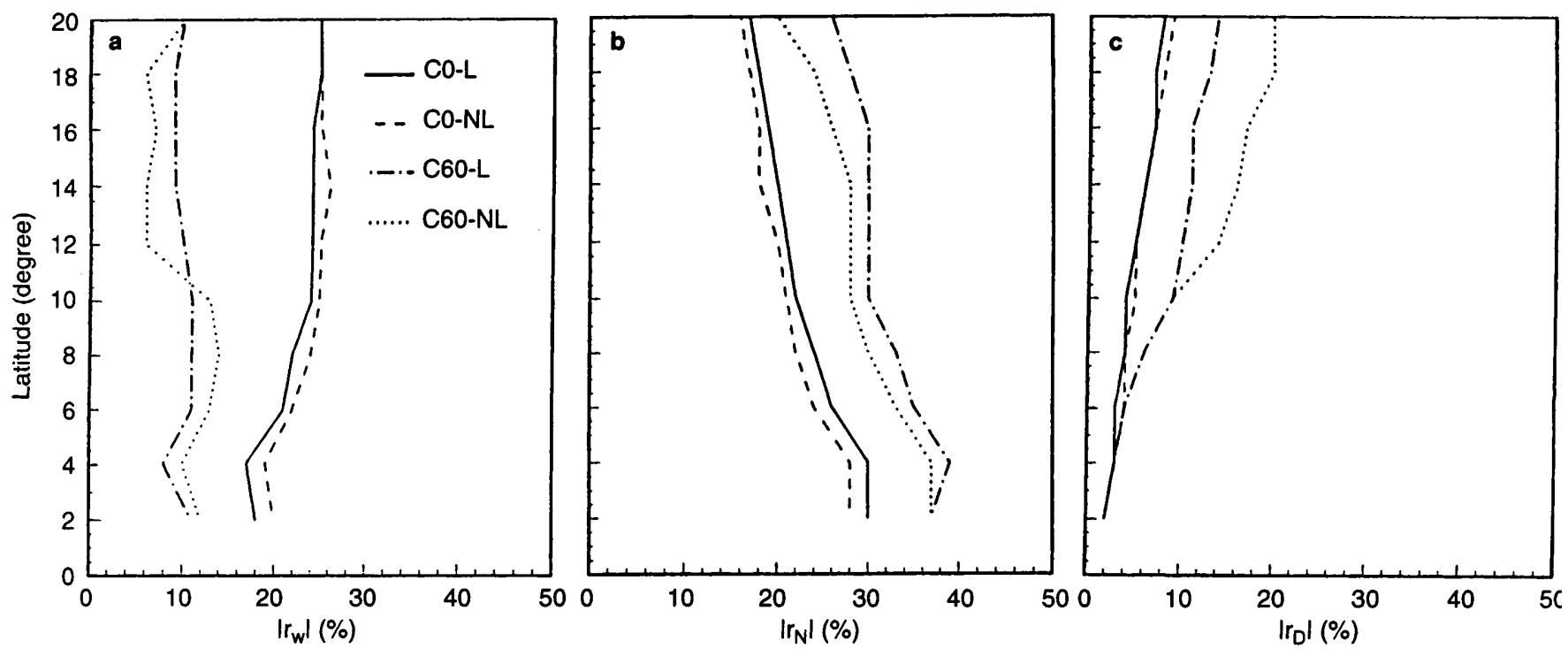

Fig. 8a-c. Energy flux coefficients $(\%)$ at different latitudes $\left({ }^{\circ}\right)$. (a) $r_{W}$, (b) $r_{N}$ and (c) $r_{D}$. Solid and center-dashed lines for the linear experiment at $\mathrm{C} 0$ and C60, respectively. Dashed and dotted lines for the non linear experiment at C0 and C60, respectively. T=60 days

The energy flux coefficients, $r_{W}, r_{N}$ and $r_{D}$, for $\mathrm{C} 0$ and C60 are shown in Fig. 10. The poleward energy flux decreases and the dissipation of energy increases as the latitude increases in both $\mathrm{C} 0$ and $\mathrm{C} 60$. The westward energy flux increases with the latitude until near $\theta_{c}$ (indicated in Fig. 9 by an arrow). As expected by the inviscid theory, above $\theta_{c}, r_{W}$ remains roughly constant with the latitude ( $r_{W} \sim 20 \%$ for $\mathrm{C} 0$ and $r_{W} 4 \%$ for C60).

The sloping boundary is less reflective than the meridional boundary (Fig. 9a) and as a result, the poleward energy flux generated at C60 is bigger than that generated at $\mathrm{C} 0$ (Fig. 9b). Figure 9c shows that more energy is dissipated in the non meridional boundary than in the meridional one. These results are qualitatively similar to those generated by $\mathrm{T}=60$ days.

The numerical (viscous) and analytical (inviscid) solutions of $r_{W}$, displayed in Table 7, show a large discrepancy between those values. The numerical results of $r_{W}$ are now much smaller than the analytical ones. Two main factors contribute to the observed difference.

The first and the most important is the inclusion of viscosity in the numerical model. The energy dissipation increases considerably as the latitude increases (Fig. 9c). Thus the energy lost in the domain certainly restrains the propagation of Rossby waves, decreasing the values of $r_{W}$.

Second, C92 obtained $r_{W}$ using the equatorial $\beta$-plane approximation whereas in the present study $r_{W}$ was obtained using spherical coordinates. The validity of this approximation to motions of a large latitudinal scale is

Table 6. Critical latitude values $\left(\theta_{c}\right)$ for the meridional $\left(\gamma=0^{\circ}\right)$ and non meridional $\left(\gamma=60^{\circ}\right)$ boundaries. $T=180$ days

\begin{tabular}{ll}
\hline & $\theta_{\mathrm{c}}\left({ }^{\circ}\right)$ \\
\hline$\gamma=0^{\circ}$ & 25.5 \\
$\gamma=60^{\circ}$ & 13.4 \\
\hline
\end{tabular}

questionable. At higher latitudes the equatorial $\beta$-plane dynamics allow an "anomalous" generation of Rossby waves, as shown in Fig. 10. The difference between the induced planetary vorticity change, given by $(\beta \Delta y)$ in the equatorial $\beta$-plane domain and by $(\Delta f)$ on the spherical coordinate domain is displayed as a function of latitude. The curve was plotted considering a constant latitudinal interval of $1^{\circ}$. As can be seen in Fig. 10, near $45^{\circ}$ of latitude the planetary vorticity change generated in the equatorial $\beta$-plane is around $30 \%$ larger than that generated using spherical coordinates. This suggests that at higher latitudes the equatorial $\beta$-plane dynamics allows a spurious generation of Rossby waves. The use of the equatorial $\beta$-plane approximation by $\mathrm{C} 92$ in the resolution of the analytical problem could help to explain the discrepancy between the numerical and analytical results obtained in the experiments. Therefore, a spherical coordinate system rather than an equatorial $\beta$-plane must be used when the north-south scale of motion is large enough for variations in the value of $\beta$ to be important.

\subsection{Annual variability}

In this experiment an equatorial Kelvin wave of annual period $(\mathrm{T}=360$ days) is used as initial condition. Table 8 shows the $\theta_{c}$ values for the meridional and sloping coastlines. For the $\mathrm{C} 0$ case $\theta_{c}$ is very close to the northern latitude of the numerical model $\left(45^{\circ}\right)$. Consequently, for the domain considered, most of the energy of the incoming equatorial wave of annual period is expected to be reflected as westward Rossby waves.

The poleward energy flux and the dissipation of energy flux were calculated using Eqs. (14) and (18) as in the other experiments. The fluxes were computed after eight months of numerical integration. Since only half a 

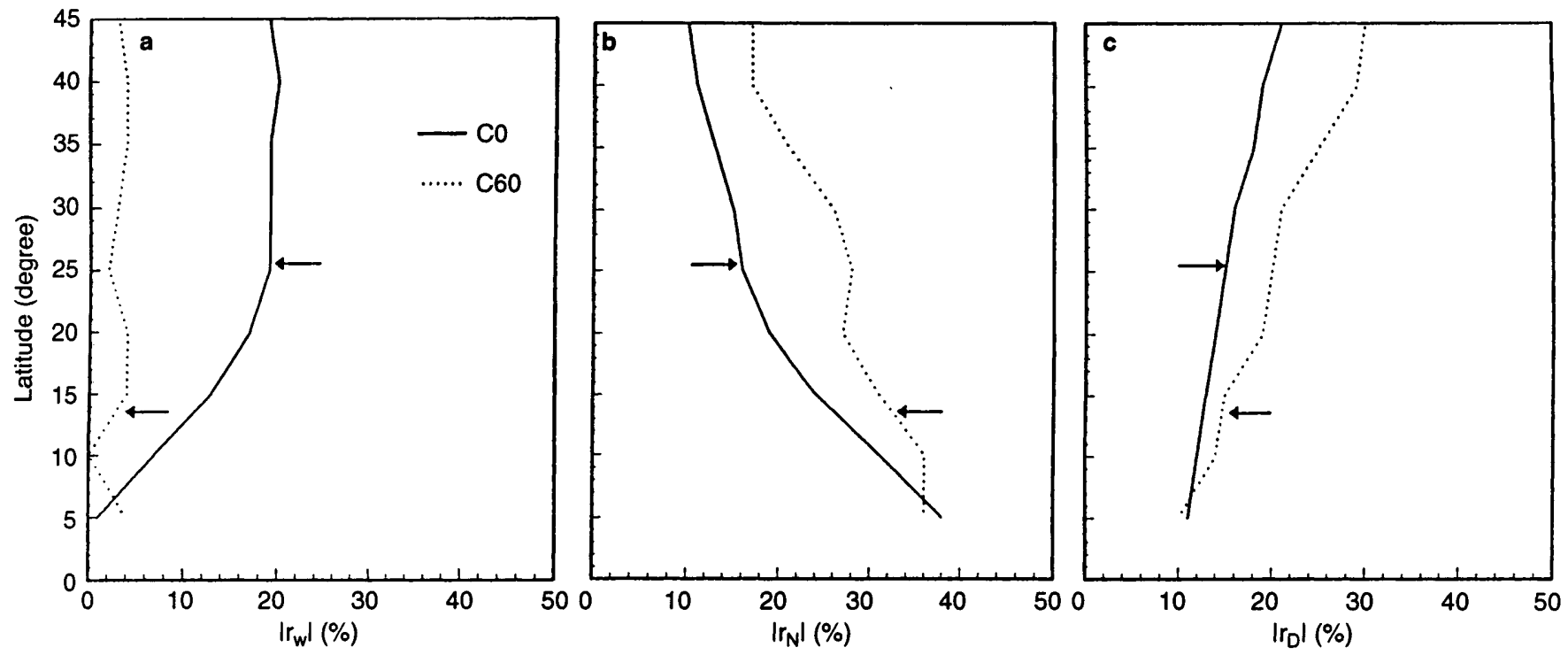

Fig. 9a-c. Linear energy flux coefficient $(\%)$ at different latitudes $\left({ }^{\circ}\right)$. (a) $r_{W}$, (b) $r_{N}$ and (c) $r_{D}$. Solid line for C0. Dotted line for C60. The arrows indicate the critical latitude values for the investigated boundaries. $\mathrm{T}=180$ days

wavelength is used as initial condition, it takes approximately 7 months of integration period for all the poleward energy to leave the numerical domain.

For both, $\mathrm{C} 0$ and $\mathrm{C} 60$, the $\mathrm{r}_{\mathrm{D}}$ values increase and the $\mathrm{r}_{\mathrm{N}}$ values decrease as the latitude increases (Fig.11), which is in qualitative agreement with theory.

Compared with the previous experiments, the westward energy fluxes present an anomalous behavior in both meridional and non meridional cases. For the meridional boundary experiment the $r_{W}$ coefficient increases latitudinally up to $30^{\circ}$ of latitude (Fig. 11a). Above latitude $30^{\circ} r_{W}$ becomes nearly constant with latitude. According to theory the $r_{W}$ coefficient is expected to be latitudinally constant only above $\theta_{c}$. Further, in the slanted boundary experiment, the $r_{W}$ coefficient is not constant with the latitude above $\theta_{c}$. It increases up to latitude $20^{\circ}$ and decreases for latitudes higher than $20^{\circ}$. The $r_{W}$ value is expected to increase up to $\theta_{c}$ and to be constant above that latitude $\left(r_{W} \sim 42 \%\right.$ from Eq. 9).

In summary, the numerical values of $r_{W}$ for $\mathrm{T}=360$ days are smaller than the theoretical coefficients. In the meridional boundary experiment, below $\theta_{c}$, the $r_{W}$ values are constant with latitude, whereas theoretically they should increase as the latitude increases. For the inclined boundary, above $\theta_{c}, r_{W}$ decreases with the latitude and according to theory it is expected to be constant as the latitude increases.

As in $\mathrm{T}=180$ days, the energy flux lost by dissipation and the use of the equatorial $\beta$-plane approximation in

Table 7. Linear westward energy flux coefficients (in $\%$ of the incident energy flux). $\mathrm{T}=180$ days

\begin{tabular}{lll}
\hline & $r_{w}$ (model) & $r_{w}$ (Eq. 9) \\
\hline$\gamma=0^{\circ}$ & 20 & 42 \\
$\gamma=60^{\circ}$ & 4 & 33 \\
\hline
\end{tabular}

Eq. (9) could be the reason for the smaller values of $r_{W}$ obtained numerically.

The values of $r_{D}$ obtained for $\mathrm{C} 60$ are much larger than the $r_{D}$ coefficients generated at $\mathrm{C} 0$ (Fig. $11 \mathrm{c}$ ). For $\mathrm{T}=360$ days most of the energy of the incoming equatorial wave is expected to be reflected at the meridional boundary as westward Rossby waves and, as already discussed, these waves are less affected by dissipation than the coastal Kelvin waves. For C60, above $25.5^{\circ}$ the incoming energy is trapped as coastal Kelvin waves. It could explain the large discrepancy between the $r_{D}$ values created in the meridional and non meridional boundaries.

The similarity of $r_{N}$ values (Fig.11b) obtained in both studied boundaries can be justified by the larger

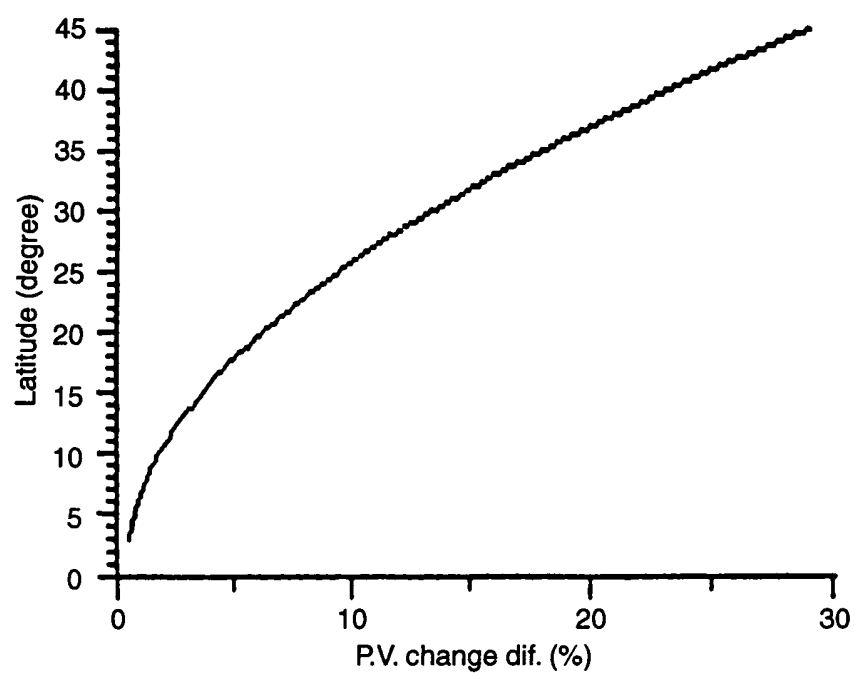

Fig. 10. Difference between the planetary vorticity change on the equatorial $\beta$-plane and on the spherical coordinates (in \%) as a function of the latitude 
Table 8. Critical latitude values $\left(\theta_{c}\right)$ for the meridional $\left(\gamma=0^{\circ}\right)$ and non meridional $\left(\gamma=60^{\circ}\right)$ boundaries. $\mathrm{T}=360$ days

\begin{tabular}{ll}
\hline & $\theta_{\mathrm{c}}\left({ }^{\circ}\right)$ \\
\hline$\gamma=0^{\circ}$ & 43.6 \\
$\gamma=60^{\circ}$ & 25.5 \\
\hline
\end{tabular}

dissipative effect in the C60 experiment (Fig.11c). The increase in $r_{N}$ induced by the coastline inclination is compensated by the increase of viscosity, resulting in similar values for $r_{N}$ in $\mathrm{C} 0$ and $\mathrm{C} 60$.

\section{Conclusions}

The main conclusions are:

1. The differences between the analytical and the numerical energy fluxes are primarily due to the inclusion of dissipative effects in the numerical model. The energy fluxes, in this model, are found to be strongly dependent on viscosity. The viscosity, however, is imperative in the model in order to form a viscous boundary layer which is required to improve agreement with observations. To a lesser degree other factors are also suggested to reinforce the discrepancy obtained between these results. It is found that the equatorial $\beta$-plane approximation in the theoretical treatment (as given by C92) can allow an artificial generation of Rossby waves at higher latitudes. The analytical theory for the inviscid case breaks down for coastline angles greater than $40^{\circ}$ in the intraseasonal case.

2. Within the confines of this model, the nonlinear effects can be regarded as negligible whereas the viscous effects are considered important.

3. For a given eastern boundary, the amount of energy dissipated in the domain is shown to be depen- dent on the wave period in two conflicting ways. Longer wave periods result in higher critical latitudes and a reduction in the presence of coastal Kelvin waves in the domain. Thus, the dissipation of energy decreases as the wave period increases. The amount of energy dissipated by the coastal Kelvin waves, however, increases as the period of the wave increases, which is in agreement with Davey et al. (1983). The final amount of energy taken from the domain by dissipation, therefore, depends not only on the presence of the coastal Kelvin waves, in the domain, but also on the period of these coastal waves.

4. The influence of the coastline geometry and the incident wave period, was found to be more important for the westward energy flux than for the poleward flux. In agreement with inviscid theory, the sloping boundaries were found to be less reflective than the meridional ones. According to the inviscid theory, the westward energy flux should increase with the frequency of the incident equatorial wave. For the viscous case this result is not always verified. The intraseasonal reflected energy, for example, is found to be larger than for those of lower periods, which is explained by the comparatively smaller values of energy dissipated in $T=60$ days.

\section{Critique}

The numerical model used in this work is very simple and omits some potentially important factors which may influence poleward wave propagation, such as higher baroclinic modes, continental shelf topography and poleward variation of the thermocline (Clarke and Gorder, 1994; Christensen et al., 1983). The importance of including these factors was not overlooked. The exclusion was based more upon computational cost than upon physical reasons.

When $A_{H}=0$ the agreement between the numerical simulations and the analytical results is absolute. The
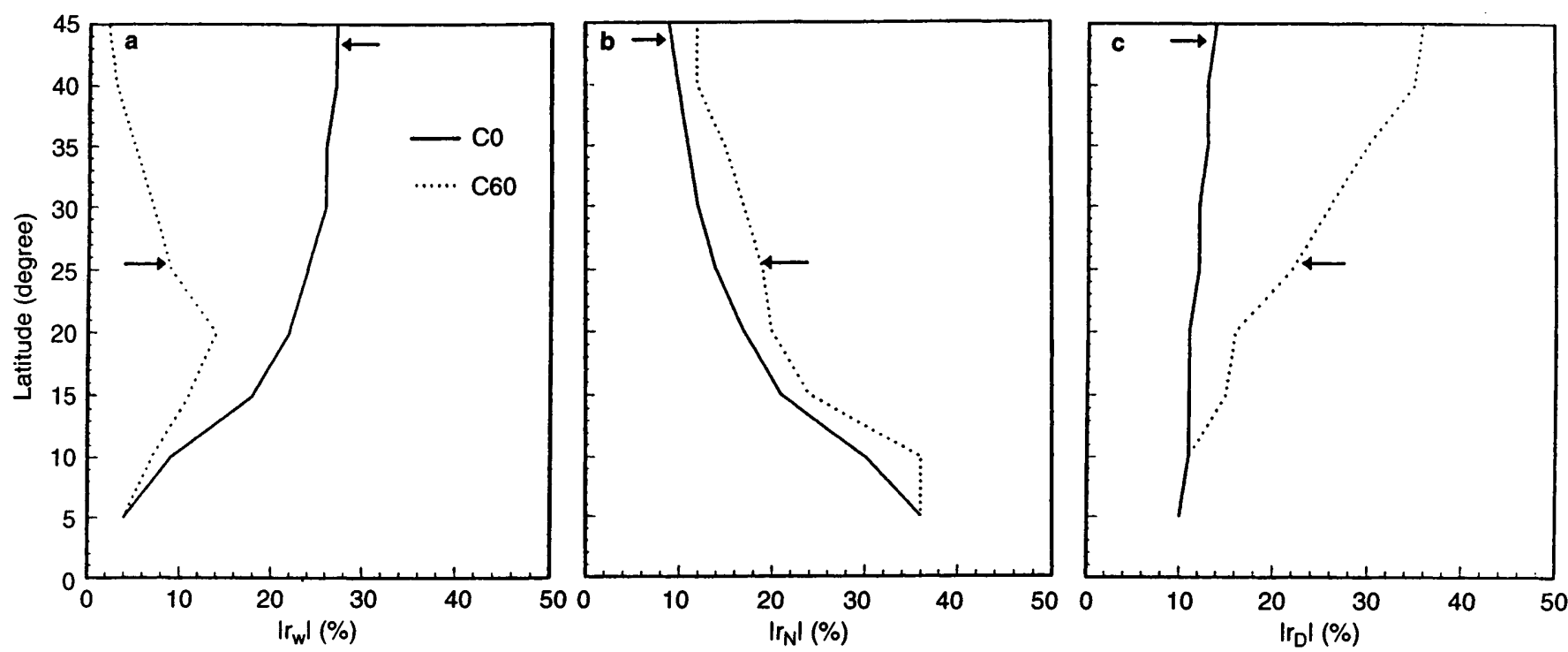

Fig. 11a-c. Linear energy flux coefficient $(\%)$ at different latitudes $\left({ }^{\circ}\right)$. (a) $r_{W}$, (b) $r_{N}$ and (c) $r_{D}$. Solid line for C0. Dotted line for C60. The arrows indicate the critical latitude values for the investigated boundaries. $\mathrm{T}=360$ days 
real ocean, however, is viscous. This study utilizes a viscosity coefficient of $10^{3} \mathrm{~m}^{2} \mathrm{~s}^{-1}$, which is a standard value widely used in models similar to this one. However, the question of how realistic this value is has yet to receive a conclusive answer.

This work assumes that the energy flux due to Kelvin waves can be separated from the one due to Rossby waves. However, in the non linear frame this is not necessarily true.

Despite the model limitations, it is expected that its numerical results will be useful for interpreting the response of more sophisticated models as well as an aid in understanding observations. The behavior of the low-frequency motion propagation from lower to higher latitudes along the eastern oceanic boundaries, however will only be completely understood through an interplay between modeling and observation. Models must be constrained by comprehensive data sets, making coincident equatorial and coastal data sets imperative for the studied problem.

Acknowledgements. J. Soares wishes to thank the Conselho Nacional de Desenvolvimento Científico e Tecnológico, CNPQ (Brasilia, Brazil) for financial support through scholarship 260102/ 89.0. Part of this work was supported by grant FAPESP 93/1387-0.

Topical Editor D. J. Webb thanks G. Bigg and another referee for their help in evaluating this paper.

\section{Appendix}

\section{Finite-difference equations}

In order to translate Eqs. (2)-(4) into finite-difference form a Richardson lattice (also known as Arakawa "C" grid) was chosen. The choice of the $\mathrm{C}$ grid is based on the fact that it best simulates the geostrophic adjustment mechanism (Mesinger and Arakawa, 1976).

The discretization scheme used in this model is a modification of the traditional forward backward (FB) scheme (Castro, 1985): the gravity wave terms, which are the layer interface displacement gradient in the momentum equations and divergent term in the continuity equation of Eqs. (2)-(4) were discretized using a FB scheme.

The FB scheme is obtained by first integrating the gravity terms of either the equation of motion or of the continuity equation forward, and then those of the other equation. Mesinger and Arakawa (1976) have studied the stability and convergence of this scheme in the context of the linearized shallow-water equations. The stability limit for the time step is twice the CFL condition valid for other discretization schemes, like the leapfrog for example, which makes this scheme very attractive in terms of computing efficiency. In addition, as a two-level scheme, it has no computational mode in time.

Henry (1981) and Foreman (1984) showed, using linearized shallow water equations, that the application of the FB scheme for both the gravity wave terms and the Coriolis terms introduces phase velocity errors for the long waves present in the model. In order to avoid these phase velocity errors, generated by asymmetric treatment of Coriolis terms, the Coriolis terms were centered in time using the Adams-Bashforth (AB) scheme. Since it is desirable to have the advective terms also centered in time, both the advection and Coriolis terms are collected together and the $\mathrm{AB}$ scheme was used to center the combined terms in time.

The AB scheme used here, a second-order accurate version (Mesinger and Arakawa, 1976), is a simplified version of the original scheme, which is of fourth order of accuracy.

The viscous term was lagged in time, as usual, in order to keep the system stable. The discretized form of Eqs. (2)-(4) used in this work is:

$$
\begin{aligned}
& u(t+\Delta t)=u(t)-\Delta t\left\{\left[1.5 \mathbf{B}_{i}(t)-0.5 \mathbf{B}_{j}(t-\Delta t)\right]\right. \\
&\left.+\delta_{i} P(t+\Delta t)-A_{H} \delta^{2} u(t)\right\} \\
& \begin{aligned}
u(t+\Delta t)=v(t) & -\Delta t\left\{\left[1.5 \mathbf{B}_{i}(t)-0.5 \mathbf{B}_{j}(t-\Delta t)\right]\right. \\
& \left.+\delta_{j} P(t+\Delta t)-A_{H} \delta^{2} v(t)\right\}
\end{aligned} \\
& h(t+\Delta t)=h(t)-\Delta t\left[\delta_{i} \mathbf{U}(t)+\frac{1}{\cos \theta_{j}} \delta_{j}\left[\mathbf{V}(t) \cos \theta_{j}\right]\right]
\end{aligned}
$$

where the indexes $i$ and $j$ indicate, respectively, the zonal and meridional directions; $\mathbf{B}$ is the combination of advective and Coriolis terms, given by:

$\left.\mathbf{B}_{i}(t)=\frac{\delta_{i}}{2} \overline{\left[u^{2}(t)^{i}\right.}+\overline{v^{2}(t)^{j}}\right]-\overline{\mathbf{V}(t)^{i j}} \overline{\mathbf{Q}(t)^{j}}$
$\left.\mathbf{B}_{j}(t)=\frac{\delta_{j}}{2} \overline{\left[u^{2}(t)^{i}\right.}+\overline{v^{2}(t)^{j}}\right]+\overline{\mathbf{U}(t)^{i j}} \overline{\mathbf{Q}(t)^{j}}$

$\mathbf{Q}$, the discretized potential vorticity, is given by:

$\mathbf{Q}(\mathrm{t})=\left\{\delta_{i} v(t)-\frac{1}{\cos \theta_{j}} \delta_{j}\left[u(t) \cos \theta_{j}\right]+f\right\} / \bar{H}^{i j}$

$P$ is the pressure integral, $P=g^{\prime} h$.

$\mathbf{U}$ and $\mathbf{V}$ are the components of the flux vector, given by:

$\mathbf{U}(t)=u(t) \overline{H(t)^{i}}$

$\mathbf{V}(t)=v(t) \overline{H(t)^{j}}$

The averaging, differentiating and Laplacian operators are respectively given by (Lilly, 1965).

Averaging operators:

$\overline{a^{i}}=\frac{1}{2}[a(i+1 / 2)+a(i-1 / 2)]$

$\overline{a^{j}}=\frac{1}{2}[a(j+1 / 2)+a(j-1 / 2)]$.

Differentiating operators:

$\delta_{i} a=\frac{1}{\Delta x}[a(i+1 / 2)-a(i-1 / 2)]$

$\delta_{j} a=\frac{1}{\Delta y}[a(j+1 / 2)-a(j-1 / 2)]$. 
Laplacian operator:

$$
\begin{aligned}
\delta^{2} a= & \frac{1}{(\Delta x)^{2}}[a(i+1)+a(i-1)-2 a(i)]+\frac{1}{(\Delta y)^{2}} \\
& \times\left\{[a(j+1)-a(j)]+\frac{\cos \theta_{j-1}}{\cos \theta_{j}}[a(j-1)-a(j)]\right\}
\end{aligned}
$$

with: $\Delta x=r \cos \theta \Delta \lambda$ and $\Delta y=r \Delta \theta$.

The system of Eqs. (A1)-(A3) is in fact implicit, but, if integrated in the order (A3)-(A1)-(A2), or (A3)-(A2)(A1) it is explicit. To minimize the asymmetry of the system the equations are integrated in the order (A3)(A1)-(A2) in the odd time steps, and in the order (A3)(A2)-(A1) in the even time steps.

The discretized form of the linear equations can also be given by Eqs. (A1)-(A3) but with $\mathbf{B}, \mathbf{Q}, \mathbf{U}$ and $\mathbf{V}$, given by:

$$
\begin{aligned}
& \mathbf{B}_{i}(t)=-\overline{V(t)^{i j} Q(t)^{j}} \\
& \mathbf{B}_{j}(t)=\overline{U(t)^{i j} Q(t)^{i}} \\
& \mathbf{Q}(t)=\frac{f}{\overline{H_{0}^{i j}}} \\
& \mathbf{U}(t)=u(t) \overline{H_{0}^{i}} \\
& \mathbf{V}(t)=v(t) \overline{H_{0}^{j}} .
\end{aligned}
$$

\section{References}

Anderson, D. L. T., and P. W. Rowlands, The role of inertia-gravity and planetary waves in the response of a tropical ocean to the incidence of an equatorial Kelvin wave on a meridional boundary, J. Mar. Res., 34, 295-312, 1976.

Anderson, D. L. T., and A. M. Moore, Data assimilation, 437-463, In Proceedings of the NATO Advanced Study on Advanced Physical Oceanographic Numerical Modeling, 1985.

Castro, B. M., Subtidal response to wind forcing in the South Brazil Bight during winter. PhD Thesis, Rosenstiel School of Marine and Atmospheric Science, University of Miami, USA, 1985.

Christensen, N. Jr., R. La Paz, and G. Gutierrez, A study of subinertial waves off the west coast of Mexico, Deep Sea Res., 30, 835-850, 1983.

Clarke, A. J., The reflection of equatorial waves from oceanic boundaries J. Phys. Oceanogr., 13, 1193-1207, 1983.

Clarke, A. J., Low-frequency reflection from a non meridional eastern ocean boundary and the use of coastal sea level to monitor eastern Pacific equatorial Kelvin waves J. Phys. Oceanogr., 22, 163-183,1992.

Clarke, A. J., and C. Shi, Critical frequencies at ocean boundaries, J. Geophys. Res., 96, 10-731-10-738,1991.

Clarke, A. J., and S. Van Gorder, On ENSO coastal currents and sea levels J. Phys. Oceanogr., 24, 661-680,1994.

Davey, M. K., W. W. Hsieh, and R. C. Wajsowicz, The free Kelvin wave with lateral and vertical viscosity, J. Phys. Oceanogr., 13, 2182-2190, 1983.

Enfield, D. B., El Niño, past and present, Rev. Geophys., 27, 159187, 1987.
Enfield, D. B., and J. S. Allen, On the structure and dynamics of monthly mean sea level anomalies along the Pacific coast of North and South America, J. Phys. Oceanogr., 10, 557-588, 1980.

Enfield, D. B., M. A. Cornejo-Rodrigues, R. L. Smith, and P. A. Newberger, The equatorial source of propagating variability along the Peru coast during the 1982-1983 El Niño, J. Geophys. Res., 92, 14-335-14-346,1987.

Foreman, M. G. G., A two dimensional dispersion analysis of selected methods for solving the linearized shallow water equations, J. Comp. Phys., 56, 287-323, 1984.

Gill, A. E., Atmosphere-Ocean Dynamics, Academic, San Diego, California, USA, 1982.

Grimshaw, R., and J. S. Allen, Low-frequency baroclinic waves off coastal boundaries, J. Phys. Oceanogr., 18, 1124-1143, 1988.

Henry, R. F., Richardson-Sielecki schemes for shallow-water equations, with applications to Kelvin waves, J. Comp. Phys., 41, 389-406, 1981.

Hsieh, W. W., M. K. Davey, and R. C. Wajsowicz, The free Kelvin wave in finite-difference numerical models, J. Phys. Oceanogr., 13, 1383-1397, 1983.

Johnson, M. I., and J. J. O'Brien, The northeast Pacific Ocean response to the 1982-1983 El Niño, J. Geophys. Res., 95, 71557166, 1990.

Longuet-Higgins, M., Planetary waves on a rotating sphere, Proc. R. Soc., London Ser. A, 279, 446-473, 1964.

Lilly, D. K., On the computational stability of numerical solutions of time-dependent non-linear geophysical fluid dynamics problems, Mon. Weather. Rev., 93, 11-26, 1965.

Luther, D. S., Observations of long period waves in the tropical oceans and atmosphere, PhD Thesis. Woods Hole Oceanographic Institution, $210 \mathrm{pp}, 1980$.

Madden, R. A., and P. R. Julian, Detection of a 40-50 day oscillation in the zonal wind in the tropical Pacific, J. Atmos. Sci., 28, 702-708, 1971.

Madden, R. A., and P. R. Julian, Description of global scale circulation cells in the tropics with a 40-50 day period, $J$. Atmos. Sci., 29, 1109-1123, 1972.

McCreary, J. P., Eastern tropical ocean response to changing wind systems, J. Phys. Oceanogr., 6, 632-645, 1976.

McPhaden, M. J., and B. Taft, Dynamics of seasonal and intraseasonal variability in the eastern equatorial Pacific, $J$. Phys. Oceanogr., 18, 1713-1732, 1988.

Mesinger, F. and A. Arakawa, Numerical methods used in atmospheric models, volume 1. GARP publication series, 17, world meteorological organization, 1976.

Miller, M. J., and A. J. Thorpe, Radiation conditions for the lateral boundaries of limited-area numerical models, Q.J. R. Meteorol. Soc., 107, 615-628, 1981.

Moore, D. W., Planetary-gravity waves in an equatorial ocean, PhD Thesis, Harvard University, 201 pp, 1968.

Orlanski, I., A simple boundary condition for unbounded hyperbolic flows, J. Comput. Phys., 21, 251-269, 1976.

Pares-Sierra, A., and J. J. O'Brien, The seasonal and interannual variability of the California current system: a numerical model, J. Geophys. Res., 94, 3159-3180, 1989.

Schopf, P. S., D. L. T. Anderson, and R. Smith, Beta-dispersion of low frequency Rossby waves, Dyn. Atmos. Ocean, 5, 187-214, 1981.

Shaffer., G, O.Pizarro, L. Djurfeldt, S. Salinas, and J. Rutllant, Circulation and low-frequency variability near the Chilean coast: remotely forced fluctuations during the 1991-92 El Niño, J. Phys. Oceanogr., 27, 217-235, 1997.

Soares, J., On the reflection of equatorial waves at eastern ocean boundaries, PhD Thesis, Oceanography Department, University of Southampton, UK, 1994.

Spillane, M. C., D. B. Enfield, and J. S. Allen, Intraseasonal oscillations in sea level along the west coast of the Americas, $J$. Phys. Oceanogr., 17, 313-325, 1986. 